VOL. 49 (1994) [483-488]

\title{
ORDERED COMPACTIFICATIONS WITH COUNTABLE REMAINDERS
}

\author{
D.C. KENT AND T.A. RICHMOND
}

\begin{abstract}
It is shown that if a partially-ordered topological space $X$ admits a finite-point $T_{2}$ ordered compactification, then it admits a countable $T_{2}$-ordered compactification if and only if it admits $n$-point $T_{2}$-ordered compactifications for all $n$ beyond some integer $m$.
\end{abstract}

\section{INTRODUCTION}

Countable compactifications of topological spaces have been studied in $[1,5,7,9]$. In [7], Magill showed that a locally compact, $T_{2}$ topological space $X$ has a countable $T_{2}$ compactification if and only if it has $n$-point $T_{2}$ compactifications for every integer $n \geqslant$ 1. We generalise this theorem to $T_{2}$-ordered compactifications of ordered topological spaces.

Before starting our generalisation of Magill's theorem, we recall two unpleasant facts about ordered compactifications. For the class of ordered topological spaces which allow $T_{2}$-ordered compactifications (that is, the $T_{3.5}$-ordered spaces), local compactness does not guarantee the existence of finite-point $T_{2}$-ordered compactifications (think of the reals with the usual order and discrete topology); furthermore the existence of an $n$-point $T_{2}$-ordered compactification for some $n>1$ does not guarantee the existence of a one-point $T_{2}$-ordered compactification (think of the reals with the usual order and topology). Here is our main theorem: If a $T_{3.5}$-ordered space $X$ allows a finite-point $T_{2}$-ordered compactification, then $X$ allows a countable $T_{2}$-ordered compactification if and only if there is a positive integer $m$ such that $X$ allows an $n$-point $T_{2}$-ordered compactification for every $n \geqslant m$. In case the order on $X$ is equality, the result is equivalent to Magill's theorem.

An ordered topological space, or simply an ordered space is a triple $(X, \tau, \theta)$ where $\tau$ is a topology on the set $X$ and $\theta$ is the graph of a partial order on $X$. An ordered space $(X, \tau, \theta)$ is $T_{2}$-ordered if $\theta$ is closed in the product space $X \times X$, and is $T_{3.5}$ ordered (completely regular ordered in [10]) if the following conditions are satisfied: (1) If $A \subseteq X$ is closed and $x \in X \backslash A$, then there exist continuous functions $f, g: X \rightarrow[0,1]$ with $f$ increasing, $g$ decreasing, $f(x)=g(x)=1$, and $f(a) \wedge g(a)=0$ for all $a \in A$;

Received 5th August, 1993.

Copyright Clearance Centre, Inc. Serial-fee code: 0004-9729/94 SA2.00+0.00. 
(2) If $x$ and $y$ are distinct points in $X$, then there exists a continuous monotone function $f: X \rightarrow[0,1]$ with $f(x)=0$ and $f(y)=1$. Compact $T_{2}$-ordered implies $T_{3.5}$-ordered, and $T_{3.5}$-ordered is hereditary.

An ordered compactification of $(X, \tau, \theta)$ is a compact $T_{2}$-ordered space $\left(X^{\prime}, \tau^{\prime}, \theta^{\prime}\right)$ such that $\left(X^{\prime}, \tau^{\prime}\right)$ contains $(X, \tau)$ as a dense subset, and $\theta \subseteq \theta^{\prime}$. We shall usually write $\left(X^{\prime}, \tau^{\prime}, \theta^{\prime}\right)$ simply as $X^{\prime}$. An ordered space has an ordered compactification if and only if it is $T_{3.5}$-ordered (see [4] or [10]). An ordered compactification $\left(X^{\prime}, \tau^{\prime}, \theta^{\prime}\right)$ of $(X, \tau, \theta)$ is strict if $\theta^{\prime}$ is the smallest order that makes $\left(X^{\prime}, \tau^{\prime}\right)$ an ordered compactification, that is, if $\theta^{\prime}$ is the intersection of all closed partial orders on $\left(X^{\prime}, \tau^{\prime}\right)$ that extend $\theta$.

If $X^{\prime}$ is an (ordered) compactification of $X$, the associated remainder is the subspace $X^{\prime} \backslash X$ of $X^{\prime}$. An (ordered) compactification whose remainder is finite or countably infinite is called a finite-point (ordered) compactification or a countable (ordered) compactification, respectively. A relation $\leqslant$ is defined on the set $K(X)$ of all compactifications of a topological space $X$ by $X^{*} \geqslant X^{\prime}$ if and only if there exists a continuous function $f: X^{*} \rightarrow X^{\prime}$ which leaves $X$ pointwise invariant. If $X^{*} \geqslant X^{\prime}$ and $X^{\prime} \geqslant X^{*}$ then $X^{*}$ and $X^{\prime}$ are equivalent compactifications. If we do not distinguish between equivalent compactifications, then $\leqslant$ is a partial order on $K(X)$. The set $K_{o}(X)$ of all ordered compactifications of ordered space $X$ can be partially ordered in the same manner with the only additional requirement that the projection function $f: X^{*} \rightarrow X^{\prime}$ be increasing.

If $\theta^{\prime}$ is a partial order on $X^{\prime}$, we shall write $x \leqslant^{\prime} y$ for $(x, y) \in \theta^{\prime}$. A set $B \subseteq X^{\prime}$ is increasing if $B=\left\{x \in X^{\prime}: b \leqslant x\right.$ for some $\left.b \in B\right\}$. Decreasing sets are defined dually. The discrete order on a set $X$ is $\Delta_{X}=\{(x, x): x \in X\}$.

\section{Countable Remainders}

A locally compact topological space $X$ has a two-point compactification if and only if $X$ has some compactification with disconnected remainder (for example, 6.16 in [2]). We say an ordered space $X$ is order disconnected if there exists a continuous increasing surjection $f: X \rightarrow\{0,1\}$ where $\{0,1\}$ has the discrete topology and the usual order $0<1$. While the existence of an order disconnected remainder does not imply the existence of a two-point ordered compactification (consider $\mathbf{R} \backslash\{0\}$, which has only three-point and four-point ordered compactifications), we do have the following result.

LEMma 2.1. Suppose $X^{\prime}$ is an $m$-point strict ordered compactification of $(X, \tau, \theta)$ and $X^{*}$ is a larger ordered compactification of $X$. Suppose $h: X^{*} \rightarrow X^{\prime}$ is the projection function and there exists $\alpha \in X^{\prime} \backslash X$ such that $h^{-1}(\alpha)$ is order disconnected. Then there exists a $(m+1)$-point ordered compactification $X^{\prime \prime}$ with $X^{\prime \prime} \geqslant X^{\prime}$, obtained by replacing $\alpha$ in $X^{\prime}$ by two compactification points.

Proof: Let $X^{\prime \prime}$ be the disjoint union of $X^{\prime} \backslash\{\alpha\}$ and $\{0,1\}$. Suppose 
$g: h^{-1}(\alpha) \rightarrow\{0,1\}$ is continuous, increasing, and onto. Define $f: X^{*} \rightarrow X^{\prime \prime}$ by $f(x)=h(x)$ for $x \in X^{*} \backslash h^{-1}(\alpha)$ and $f(x)=g(x)$ for $x \in h^{-1}(\alpha)$. If $X^{\prime \prime}$ is given the quotient topology $\tau^{\prime \prime}$ derived from $f$ and $X^{*}$, then $\left(X^{\prime \prime}, \tau^{\prime \prime}\right)$ is a topological compactification of $X$.

Define a relation $\theta^{\prime \prime}$ on $X^{\prime \prime}$ by $a \leqslant$ if $b$ if and only if there exist points $a=$ $c_{0}, c_{1}, \ldots, c_{n}=b$ in $X^{\prime \prime}$ such that for each $i=1, \ldots, n$, there exists a net $\left(x_{\lambda}, y_{\lambda}\right)$ in $\theta$ converging in $X^{\prime \prime} \times X^{\prime \prime}$ to $\left(c_{i-1}, c_{i}\right)$. The points $a=c_{0}, c_{1}, \ldots, c_{n}=b$ are called a trail from $a$ to $b$ with length $n$. In [12, Theorem 1.1] it is shown that the analogous relation $\leqslant^{\prime}$ defined on $X^{\prime}$ is the strict order on $X^{\prime}$. Observe that the nets $\left(x_{\lambda}, y_{\lambda}\right)$ defining a trail are nets in $\theta \subseteq X^{2}$ and thus $\left(x_{\lambda}\right)$ and $\left(y_{\lambda}\right)$ are embedded in $X^{\prime}, X^{\prime \prime}$, and $X^{*}$. Since $X^{\prime}$ is a quotient of $X^{\prime \prime}, x_{\lambda} \rightarrow c_{i}$ in $X^{\prime \prime}$ implies $x_{\lambda} \rightarrow c_{i}^{\prime}$ in $X^{\prime}$, where $c_{i}^{\prime}=c_{i}$ if $c_{i} \in X^{\prime \prime} \backslash\{0,1\}=X^{\prime} \backslash\{\alpha\}$, and $c_{i}^{\prime}=\alpha$ if $c_{i} \in\{0,1\}$. If $c_{0}, \ldots, c_{n}$ is a trail in $X^{\prime \prime}$ from $c_{0}$ to $c_{n}$ where $c_{0}, c_{n} \in X^{\prime \prime} \backslash\{0,1\}$, then $c_{0}=c_{0}^{\prime}, c_{1}^{\prime}, \ldots, c_{n}^{\prime}=c_{n}$ is a trail in $X^{\prime}$ from $c_{0}$ to $c_{n}$, and thus $c_{0} \leqslant c_{n}$. This shows that $\theta^{\prime \prime}$ extends $\theta^{\prime} \cap\left(X^{\prime} \backslash\{0,1\}\right)^{2}$, and therefore extends $\theta$.

We now show that $\leqslant "$ is antisymmetric. Suppose $a \leqslant " b$ and $b \leqslant " a$. If $a, b \in$ $X^{\prime \prime} \backslash\{0,1\}=X^{\prime} \backslash\{\alpha\}$, then $a \leqslant^{\prime} b$ and $b \leqslant \leqslant^{\prime} a$, and thus $a=b$. If $a \in X^{\prime \prime} \backslash\{0,1\}$ and $b \in$ $\{0,1\}$, then the trails from $a$ to $b$ and from $b$ to $a$ imply $a \leqslant^{\prime} \alpha$ and $\alpha \leqslant^{\prime} a$, contrary to the fact that $a \in X^{\prime \prime} \backslash\{0,1\}=X^{\prime} \backslash\{\alpha\}$. Finally, suppose $a, b \in\{0,1\}$, that is, suppose $0 \leqslant " 1$ and $1 \leqslant " 0$. Since $0 \leqslant " 1$, there exists a trail $0=c_{0}, \ldots, c_{i}, \ldots, c_{n}=1$ in $X^{\prime \prime}$ from 0 to 1 . Viewing the nets involved as nets in $X^{\prime}$ we have $\alpha=0^{\prime} \leqslant \leqslant^{\prime} c_{i}^{\prime} \leqslant 1^{\prime} 1^{\prime}=\alpha$, and thus $c_{i} \in\{0,1\}$. Thus, the only trail with minimal length from 0 to 1 is 0,1 . Similarly, $1 \leqslant 0$ implies 1,0 is the unique minimal trail from 1 to 0 . Suppose $\left(x_{\lambda}, y_{\lambda}\right)$ is a net in $\theta$ converging to $(0,1)$ and $\left(z_{\gamma}, w_{\gamma}\right)$ is a net in $\theta$ converging to $(1,0)$. Now in $X^{*} \times X^{*}$, there are convergent subnets $\left(x_{\sigma(\lambda)}, y_{\sigma(\lambda)}\right) \rightarrow\left(a^{*}, b^{*}\right)$ and $\left(z_{\rho(\gamma)}, w_{\rho(\gamma)}\right) \rightarrow\left(b^{\#}, a^{\#}\right)$ where $a^{*}, a^{\#} \in g^{-1}(0)$ and $b^{*}, b^{\#} \in g^{-1}(1)$. Since these subnets are in $\theta$ and $\theta^{*}$ is closed, it follows that $a^{*} \leqslant \leqslant^{*} b^{*}$ and $b^{\#} \leqslant{ }^{*} a^{\#}$. But $1=g\left(b^{\#}\right) \notin g\left(a^{\#}\right)=0$, contrary to $g$ being increasing. Thus $0 \leqslant 1$ and $1 \leqslant " 0$ is not possible, and $\leqslant "$ is antisymmetric. The relation $\leqslant$ " is easily seen to be reflexive and transitive, and is thus a partial order on $X^{\prime \prime}$.

To show that $\leqslant$ " is closed in $X^{\prime \prime} \times X^{\prime \prime}$, it suffices to show that if $\left(A_{\gamma}, B_{\gamma}\right)$ is any net in $\leqslant$ " converging to $(A, B)$, then $A \leqslant$ " $B$. This can be shown by an induction argument on $\max _{\gamma}\left\{\right.$ length of a minimal trail from $A_{\gamma}$ to $\left.B_{\gamma}\right\}$ (which is bounded), as in the proof of Theorem 1.1 of [12].

Thus, $\left(X^{\prime \prime}, \tau^{\prime \prime}, \leqslant^{\prime \prime}\right)$ is a strict ordered compactification of $X$ with $X^{\prime \prime} \geqslant X^{\prime}$.

The lemma below gives us a supply of order disconnected spaces.

LEMMA 2.2. Every countable $T_{3.5}$-ordered space is order disconnected. 
ProOF: We shall show the stronger result that for any distinct points $x$ and $y$ in a countable $T_{3.5}$-ordered space $X$, there exists a continuous increasing surjection $g: X \rightarrow\{0,1\}$ with $g(x) \neq g(y)$. Let $C I^{*}(X)$ denote the set of continuous increasing functions from $X$ to $[0,1]$. Since $X$ is $T_{3.5}$-ordered, the evaluation map $e: X \rightarrow$ $[0,1]^{C I^{*}(X)}$ defined by $e(x)=\Pi_{f \in C I^{*}(X)} f(x)$ is a topological and order embedding (see [4]). Choose $f_{0} \in C I^{*}(X)$ such that $f_{0}(x) \neq f_{0}(y)$. Since $X$ is countable, there exists an irrational number $\alpha$ strictly between $f_{o}(x)$ and $f_{o}(y)$ with $\alpha \notin \pi_{f_{o}}(e(X))$. Now since the projection $\pi_{f_{o}}$ is continuous and increasing, $\pi_{f_{o}}^{-1}([0, \alpha))=\pi_{f_{o}}^{-1}([0, \alpha])=U$ is a closed, open, decreasing set in $e(X) \approx X$. The function $g: X \rightarrow\{0,1\}$ defined by $g(U)=0$ and $g(X \backslash U)=1$ has the desired properties.

In [3] Engelking and Sklyarenko show that the supremum of a set $\left\{X_{i}\right\}_{i \in I}$ of compactifications of a topological space $X$ can be constructed by forming the product $P=\Pi_{i \in I} X_{i}$, identifying $X$ with the subspace $\left\{z \in P: z=\Pi_{i \in I} x\right.$ for some $x \in$ $X\}$, then taking $c l_{P} X$. This construction also yields the supremum of any set of ordered compactifications. By 1.8 of [8], the remainder of the supremum of a set of (ordered) compactifications is contained in the product of the remainders of these (ordered) compactifications. Thus, we have the following result.

LEMMA 2.3. If $\left\{X_{i}\right\}_{i \in I}$ is a set of (ordered) compactifications of $X$ with $\left|X_{i} \backslash X\right|<\rho$ for each $i \in I$, then $\sup \left\{X_{i}\right\}_{i \in I}$ is an (ordered) compactification whose remainder has cardinality at most $\rho \times|I|$.

Theorem 2.4. Suppose $(X, \tau, \theta)$ admits finite-point ordered compactifications. Then $X$ has a countable ordered compactification if and only if $X$ admits $n$-point ordered compactifications for all integers $n$ greater than some $m$.

ProOF: Suppose $X$ has $m$-point ordered compactification $X^{\prime}$ and countable ordered compactification $X^{*}$. Without loss of generality, we may assume $X^{\prime}$ is a strict ordered compactification, and $X^{*} \geqslant X^{\prime}$ (otherwise, replace $\theta^{\prime}$ by the strict order on $\left(X^{\prime}, \tau^{\prime}\right)$ and replace $X^{*}$ by $\left.\sup \left\{X^{\prime}, X^{*}\right\}\right)$. If $h: X^{*} \rightarrow X^{\prime}$ is the projection function, there must exist $\alpha \in X^{\prime} \backslash X$ such that $h^{-1}(\alpha)$ is countable. By Lemmas 2.2 and 2.1, $X$ has an $(m+1)$-point ordered compactification $X^{\prime \prime}$. Repeating this process shows that $X$ has $n$-point ordered compactifications for all $n \geqslant m$.

Conversely, if $X$ admits $n$-point ordered compactifications $X_{n}$ for all $n>m$, Lemma 2.3 implies that $\sup \left\{X_{n}: n>m\right\}$ is a countable ordered compactification of $\boldsymbol{X}$.

THEOREM 2.5. If $(X, \tau, \theta)$ admits a countable ordered compactification $X^{*}$ and a finite-point ordered compactification $X^{\prime}$ with $X^{\prime} \leqslant X^{*}$, then $X^{*}$ is the supremum of all finite-point ordered compactifications below it.

Proof: The proof is analogous to that of Theorem 2.3 of [9]. Let $X^{\prime \prime}=\sup \left\{X^{\#} \leqslant\right.$ 
$X^{*}: X^{\#}$ is a finite-point ordered compactification of $\left.X\right\}$. Clearly $X^{*} \geqslant X^{\prime \prime}$. Equality holds if the projection $f: X^{*} \rightarrow X^{\prime \prime}$ is one-to-one. Suppose $x \neq y$ in $X^{*}$. Is the projection $h: X^{*} \rightarrow X^{\prime}$ maps $x$ and $y$ to distinct points, then $f(x) \neq f(y)$. If $h(x)=h(y)$, use the strong statement proved in Lemma 2.2 to find a finite-point ordered compactification $X^{\#} \leqslant X^{*}$ such that the projection $k: X^{*} \rightarrow X^{\#}$ does separate $x$ and $y$.

THEOREM 2.6. Suppose $X$ admits a finite-point ordered compactification. Then $X$ has a largest finite-point ordered compactification if and only if it has no countable ordered compactification.

Proof: If $X$ has no countable ordered compactification, then there is an integer $n$ such that $X$ has an $n$-point ordered compactification but no $m$-point ordered compactifications for $m>n$. Now any two $n$-point ordered compactifications must be topologically equivalent, for otherwise by considering the associated $n$-stars (see [6]) we find that the supremum of the topological compactifications underlying the two $n$-point ordered compactifications has more than $n$ compactification points. Now by the remarks preceeding Lemma 2.3, the supremum of a set of ordered compactifications is topologically equivalent to the supremum of the set of underlying topological compactifications, which leads to the contradition that $X$ admits an $m$-point ordered compactification with $m>n$. Thus, all $n$-point ordered compactifications of $X$ are topologically equivalent; intersecting their orders gives a largest finite-point ordered compactification.

The converse is immediate from Theorem 2.4.

Although Theorem 2.6 gives necessary and sufficient conditions for the existence of a largest finite-point ordered compactification, no such result is known which guarantees the existence of a smallest ordered compactification, finite-point or otherwise. Indeed, if $X$ is the half-open interval $[0,1)$ with the usual topology and discrete order, there is a unique largest finite-point ordered compactification whose order is also discrete, however there is no smallest ordered compactification of $X$.

If a $T_{3.5}$-ordered space $X$ admits a finite-point ordered compactification, it obviously admits ordered compactifications whose remainders have minimal finite cardinality; we call any such compactification a minimal-point ordered compactification. If $X$ has a smallest finite-point ordered compactification, then all minimal-point ordered compactifications of $X$ have equivalent topologies, but the converse is false as is shown by the example of the preceding paragraph. On the other hand, if all minimalpoint ordered compactifications of $X$ have equivalent order, there exists a smallest ordered compactification; again, the converse is false. In general, minimal point ordered compactifications of the same space may have non-equivalent topologies and/or non-equivalent order. 
Finally, for the sake of comparing finite-point ordered compactifications with finitepoint (non-ordered) compactifications, we mention a few additional facts. A $T_{3.5^{-}}$ ordered space may have a largest finite-point (non-ordered) compactification but no largest finite-point ordered compactification (for example, the Euclidean plane); on the other hand, it may have a largest finite-point ordered compactification but no largest finite-point (non-ordered) compactification (for example, the natural numbers). There are also examples of $T_{3.5}$-ordered spaces which have a largest finite-point ordered compactification and a largest finite-point (non-ordered) compactification whose remainders are of different cardinality.

\section{REFERENCES}

[1] G.L. Cain, 'Countable compactifications', in General topology and its relations to modern analysis and algebra VI, (Z. Frolík, Editor), Proc. Sixth Prague Topological Symposium 1986 (Heldermann Verlag, Berlin, 1988), pp. 69-75.

[2] R.E. Chandler, Hausdorff compactifications, Lecture Notes in Pure and Appl. Math. 23 (Marcel Dekker, Inc., New York, 1976).

[3] R. Engelking and E.G. Sklyarenko, 'On compactifications allowing extensions of mappings', Fund. Math. 53 (1963), 65-79.

[4] P. Fletcher and W. Lindgren, Quasi-uniform spaces, Lecture Notes in Pure and Appl. Math. 77 (Marcel Dekker, Inc., New York, 1982).

[5] T. Kimura, ' $\aleph_{0}$-point compactifications of locally compact spaces and product spaces', Proc. Amer. Math. Soc. 93 (1985), 164-168.

[6] K.D. Magill, Jr., ' $N$-point compactifications', Amer. Math. Monthly 72 (1965), 1075-1081.

[7] K.D. Magill, 'Countable compactifications', Canad. J. Math. 18 (1966), 616-620.

[8] J.R. McCartney, 'Maximum zero-dimensional compactifications', Math. Proc. Cambridge Philos. Soc. 68 (1970), 653-661.

[9] J.R. McCartney, 'Maximum countable compactifications of locally compact spaces', Proc. London Math. Soc. (3) 22 (1971), 369-384.

[10] L. Nachbin, Topology and order, New York Math. Studies 4 (Van Nostrand, Princeton, N.J., 1965).

[11] T.A. Richmond, 'Finite-point order compactifications', Math. Proc. Cambridge Philos. Soc. 102 (1987), 467-473.

[12] T.A. Richmond, 'Posets of ordered compactifications', Bull. Austral. Math. Soc. 47 (1993), 59-72.

Department of Pure and Applied Mathematics

Washington State University

Pullman, WA 99164-3113

United States of America
Department of Mathematics

Western Kentucky University

Bowling Green, KY 42101

United States of America 